\title{
Best practice of nutritional support for pediatric acute pancreatitis
}

\author{
Wei Cai ${ }^{1}$
}

Published online: 23 September 2021

(c) Children's Hospital, Zhejiang University School of Medicine 2021

In this issue of World Journal Pediatrics, Zhao et al. address that nutrition support by nasogastric (NG) or nasojejunal (NJ) feeding has similar results in pediatric acute pancreatitis (AP) [1]. AP in children is one of the common acute abdominal diseases in childhood, and the incidence is increasing significantly in recent decades [2]. Nutritional support is one of the key points for AP patients' treatment. Compared with fasting or parenteral nutrition, published papers from developed countries had showed that an early start of enteral nutrition through $\mathrm{NG}$ and $\mathrm{NJ}$ tube, especially within 48 hours, is beneficial to shorten the length of hospital stay for AP management, which was common sense in AP [3]. However, the current recommendations of guidelines or common sense for the treatment of AP in children are based primarily on evidence in adults, and there is a lack of children-oriented research, especially prospective randomized controlled studies. On the other hand, previous studies have reported that NG feeding is not inferior to $\mathrm{NJ}$ feeding [4]. A prospective randomized controlled trial was first designed by Zhao et al. to compare the effects of NG feeding and NJ feeding in treating AP in children. The authors demonstrated that the NG tube and the NJ tube had similar tolerance, and the NG tube could shorten the tube feeding time and the length of hospital stay. This study concluded that NG feeding and NJ feeding were equivalently effective and safe in the treatment for mild-to-moderate AP in children, which provided positive evidence for enteral nutrition therapy for AP in children. These new findings may change routine clinical practice in pediatric AP because NG offers easier placement and is closer physically. However, the number of cases from this study was not high enough,

Wei Cai

caiw1978@163.com

1 Division of Pediatric Gastroenterology and Nutrition, Department of Pediatric Surgery, Xinhua Hospital, Shanghai Jiao Tong University, School of Medicine, Shanghai 200092, China and a multi-center randomized controlled trial was needed for stronger evidence in comparing alternative methods of enteral nutrition for mild-to-moderate pediatric AP patients. Ultimately, the possibility of using early oral intake for mild AP also needs further study to provide evidence in children.

Author contributions Wei Cai is the only author of this commentary.

Funding None.

\section{Compliance with ethical standards}

Ethical approval Not required.

Conflict of interest The author declared no financial or non-financial conflict of interest related to this article. Dr. Wei Cai is a member of the Editorial Board for World Journal of Pediatrics. The paper was handled by the other Editor and has undergone rigrous peer review process. Dr. Wei Cai was not involved in the journal's review of, or decisions related to, this manuscript.

\section{References}

1. Zhao H, Han Y, Peng KR, Luo YY, Yu JD, Fang YH, et al. Nasogastric or nasojejunal feeding in pediatric pancreatitis: a randomized controlled trial. World J Pediatr. 2021. https://doi. org/10.1007/s12519-021-00441-0.

2. Pant C, Deshpande A, Olyaee M, Anderson MP, Bitar A, Steele MI, et al. Epidemiology of acute pancreatitis in hospitalized children in the United States from 2000-2009. PLoS ONE. 2014;9:e95552.

3. Feng P, He C, Liao G, Chen Y. Early enteral nutrition versus delayed enteral nutrition in acute pancreatitis: a PRISMA-compliant systematic review and meta-analysis. Medicine (Baltim). 2017;96:e8648.

4. Zhu Y, Yin H, Zhang R, Ye X, Wei J. Nasogastric nutrition versus nasojejunal nutrition in patients with severe acute pancreatitis: a meta-analysis of randomized controlled trials. Gastroenterol Res Pract. 2016;2016:6430632.

Publisher's Note Springer Nature remains neutral with regard to jurisdictional claims in published maps and institutional affiliations. 